\title{
Synthesis, Electrochemistry, STM Investigation of Oligothiophene Self-Assemblies with Superior Structural Order and Electronic Properties
}

Cheng-Yu Kuo, ${ }^{1}$ Yinghao Liu, ${ }^{2}$ Dmitry Yarotski, ${ }^{2}$ Hao Li, ${ }^{3}$ Ping Xu, ${ }^{1}$ Hung-Ju Yen, ${ }^{1}$ Sergei Tretiak, ${ }^{3 *}$ Hsing-Lin Wang, ${ }^{1 *}$

${ }^{1}$ C-PCS, Chemistry Division, ${ }^{2}$ Center of Integrated Nanotechnologies, Materials Physics and Application Division, ${ }^{3}$ Theory Division, Los Alamos National Laboratory, Los Alamos, New Mexico, 87545

\begin{abstract}
:
Three oligothiophene (terthiophene, tetrathiophene and pentathiophene) derivatives are synthesized and their monolayer self-assemblies on gold ( $\mathrm{Au}$ ) are prepared via Au-S covalent bond. Our UV-Vis experimental characterization of solution reveals the dependence of the optical properties on the conjugation length of the oligothiophenes, which compares well with Time-Dependent Density Functional Theory (TDDFT) simulations of spectra of individual chromophores. Photoluminescent spectra of thin films show pronounced red shifts compared to that of solutions, suggesting strong inter-oligomer interactions. The comparative studies of cyclic voltammograms of tetrathiophene from solution, cast film and self-assembled monolayer (SAM) indicate presence of one, two, and three oxidized species in these samples, respectively, suggesting a very strong electronic coupling between tetrathiophene molecules in the SAM. Scanning tunneling microscopy (STM) imaging of SAMs of the tetrathiophene on an atomically flat $\mathrm{Au}$ surface exhibits formation of monolayer assemblies with molecular order, and the molecular packing appears to show an overlay of oligothiophene molecules on top of another one. In contrast, the trimer and pentamer images show only aggregated species lacking longrange order on the molecular level. Such trends in going from disordered-ordered-disordered monolayer assemblies are mainly due to a delicate balance between inter-chromophore $\pi-\pi$ couplings, hydrophobic interaction and the propensity to form Au-S covalent bond. Such hypothesis has been validated by our computational results suggesting different interaction patterns of oligothiophenes with odd numbered and even numbered thiophene repeat units placed in a dimer configuration. Observed correlations between oligomer geometry and structural order of monolayer assembly elucidate important structure-property relationships and have implications for these molecular structures in organic optoelectronic devices and energy devices.
\end{abstract}

Keywords: oligothiophene; self-assembly; spectroscopy; structural order; TDDFT 


\section{Introduction:}

Conjugated oligomers have attracted lots of attention due to their potential applications in sensing [1], electronic [2] and optical [3] technologies. Of particular interest are oligothiophenes and their derivatives that have been studied extensively in the last decade due to potential applications in molecular electronics [3a, 4]. The oligothiophene-based thin film transistors [5] and sensing devices reveal properties that are strongly dependent on the packing of molecules spanning various hierarchical 2D and 3D architectures [6]. Interestingly, the packing between oligothiophenes have been shown to be temperature- and conformational-dependent [7]. The variation of the optical and transport properties of oligothiophene thin films is dominated by the way molecules come together (packing) that can be achieved over a large area through molecular self-assembly [8]. Self-assemble monolayer (SAM) of conjugated oligomers through covalent bond between thiolated functional group and single crystal Au (111) substrate [9] thioacetylated functional group on $\mathrm{Au}$ [10], and siloxane terminated conjugated oligomer on $\mathrm{SiO}_{2}$ surface [11] have been demonstrated. More recently, the use of such self-assembled monolayers (SAM) through Langmuir Blodgett method [12], consisting of conjugated polymers/oligomers have found broad applications in controlling and modifying the donoracceptor interfaces for improved power conversion efficiencies [13]. To better understand the structure-property relationships in oligothiophene SAMs, we have synthesized a series of thiolated conjugated oligomers (oligothiophenes) that can be covalently bound to atomically flat $\mathrm{Au}$ substrates. We apply STM and AFM microscopic probes to determine how the molecular structure and binding geometry of the covalent end groups to the Au surface impact the optical and redox properties of conjugated oligomer SAMs. Complementary electronic structure simulations provide detailed insights into nature of essential excited states, optical spectra and allow to rationalize experimental trends. Our results provide detailed insights into the mechanisms underpinning SAM functionality that are crucial for future applications in designing functional molecules for molecular electronics, field-effect transistors (FET) and photovoltaic devices.

This article is organized as follows: Section II briefly introduces synthesis, experimental characterization and computational approaches used in this study. In Section III, we describe in detail main results for properties of individual oligothophenes, their aggregates and selfassemblies. Finally, Section IV summarizes our findings and provides conclusions and possible future directions.

\section{Methods:}

\section{Synthesis}

Chemical structures of oligothiophene derivatives are shown in the top panel of Figure 1; all the oligothiophenes have the same thiolated end group for binding to the Au substrate and an alkyl head group to improve solubility in organic solvents. The only difference is the conjugation length that extends from trimer to tetramer to pentamer. Synthesis of thiophene 
oligomers with thiolated end group are carried out via Stille coupling and Grignard reaction between 2bormothiophene and 2tripropylstannane thiophene. Detailed synthetic procedure is described in Scheme 1 in the supplemental information. Spectral characterization was conducted on the as-prepared solutions. The monolayers were deposited by immersing flame-annealed $\mathrm{Au}(111)$ films (SPI Supplies) into the solution for 60 minutes. The films were then extracted and rinsed with acetone to remove excess oligothiophene and immediately transferred to UHV chamber for STM study.

\section{Experimental characterization}

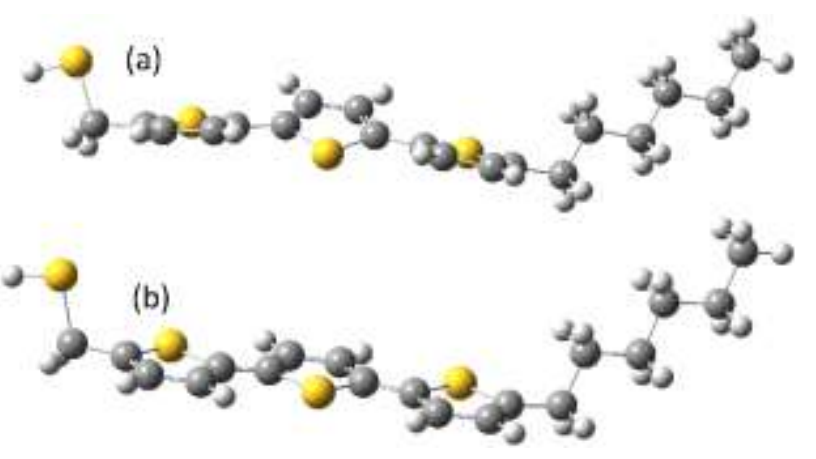

Figure 1. (Top) Molecular structures of terthiophene, tetrathiophene, pentathiophene. (Bottom) Optimized terthiophene geometries of ground (a) and excited (b) states at $\omega \mathrm{B} 97 \mathrm{XD} / 6-31 \mathrm{G}(\mathrm{d}) / \mathrm{LR}$ level.

Measurements of the molecular arrangements on the substrate surfaces were made using a commercial ultrahigh vacuum variable-temperature STM system (RHK Technology Inc., UHV750). All measurements were performed at room temperature and a pressure of $3 \times 10^{-10}$ Torr. Freshly etched tungsten tips were cleaned in situ by field emission into the gold surface before each measurement. Absorption and fluorescence measurements were performed using Varian 450 spectrophotometer and PTI fluorimeter.

\section{Quantum Chemical Calculations}

Electronic properties of the thiolated oligothiophenes were investigated using density functional theory (DFT) for ground state and time-dependent DFT (TDDFT) for excited state properties. All presented simulations were conducted with Gaussian 09 package [14]. Hybrid functional $\omega B$ 97XD [15] with 6-31G(d) basis sets was employed in all DFT/TDDFT computations associated with electronic ground and excited states in oligothiophenes. The $\omega B$ 97XD functional accounts for weak dispersive interactions due to the intermolecular $\pi-\pi$ stacking using empirical damped atom-atom dispersion parameters within Grimme's D2 model [16], in addition to the full exchange correction at long distance to describe delocalized $\pi \rightarrow \pi^{*}$ transitions. Previously, we have benchmarked this computational methodology for stacked oligothiophene systems against the results of the algebraic-diagrammatic construction approach at the second-order perturbation level (ADC(2)) [17]. The ADC(2) approach is an accurate wave function-based electron propagator method built on the many-body Green's function formalism [18]. Excellent agreements obtained in both vacuum and solutions justify the capability and 
accuracy of the TDDFT simulation of electronic excited states including low-laying CT excitations in the conjugated oligomers [17].

The absorption and emission spectra were computed in dichloromethane solution with the dielectric constant $\varepsilon=8.93$ at $25^{\circ} \mathrm{C}$. The overall spectral shape was obtained using empirical Lorentzian broadening of $0.1 \mathrm{eV}$ of oscillator strength for calculated electronic transitions [19]. The simulations in solution were conducted using the self-consistent reaction field (SCRF) approach with basic solvation model of the conductor-like polarizable continuum model (C-PCM) [20], which is numerically efficient. In addition to the spectroscopic computation under the conventional linear response (LR) model, in which the solvent reaction field is classically related to the transition density, we also conducted the simulation using the state specific (SS) solvation model [21], in which the effect of the solvent reaction field on the solute wave function is quantum mechanically accounted for. The LR model is generally limited to weak perturbation systems so that it may not be adequate for describing highly polarizable excitations in the oligomers of our interest. The SS model accounts for the solvation correction energy due to the state-dependent fast electronic relaxation of the solvent polarization, hence has its advantage over the LR approach to treat dynamical solvent effects involving in the calculation of excitation in solvent. Further details of the SS method within TDDFT framework can be found in references [21].

Further, to estimate the strength of intermolecular $\pi-\pi$ interaction, we computed the binding and separation energies of molecular dimers [22]. The binding energy describes the energy released in the process of combining two monomers in ground state into an energetically optimized dimer. In the calculation of the separation process, the two molecules in an optimized dimer configuration are manually placed far apart from each other $(>1000 \mathrm{~nm})$ without further relaxation calculation. Because the monomer geometries are not energetically optimized in the separation process, the absolution value of the separation energy is larger than that of the corresponding binding energy (Table SI). Note that the negative values denote releasing energy whereas positive sign represents energy absorption.

\section{Results and Discussions:}

Figure 2 (top, left) shows the experimental UV-Vis and photoluminescence (PL) spectra of thiolated oligothiophenes in chloroform solution. As expected, monotonic red shift in the absorbance and fluorescence spectra from trimer to pentamer is observed, consistent with previous studies of the optical spectra dependence on the conjugation length [23]. Moreover, obvious vibronic features in the fluorescence spectra suggest the dominance of single molecules in solution [24].

Calculated molecular geometries of ground and excited states are shown in the bottom panel of Figure 1. In optimized ground-state geometries of the monomers, the thiol group and the hexane chain can be positioned on either side of the oligothiophene plane because the configuration energy difference $(\sim 3 \mathrm{meV})$ is smaller than the thermal energy $k \mathrm{~T}(25 \mathrm{meV}$ at room temperature). The dihedral angle between adjacent thiophenes is about $152^{\circ}-155^{\circ}$ so that 


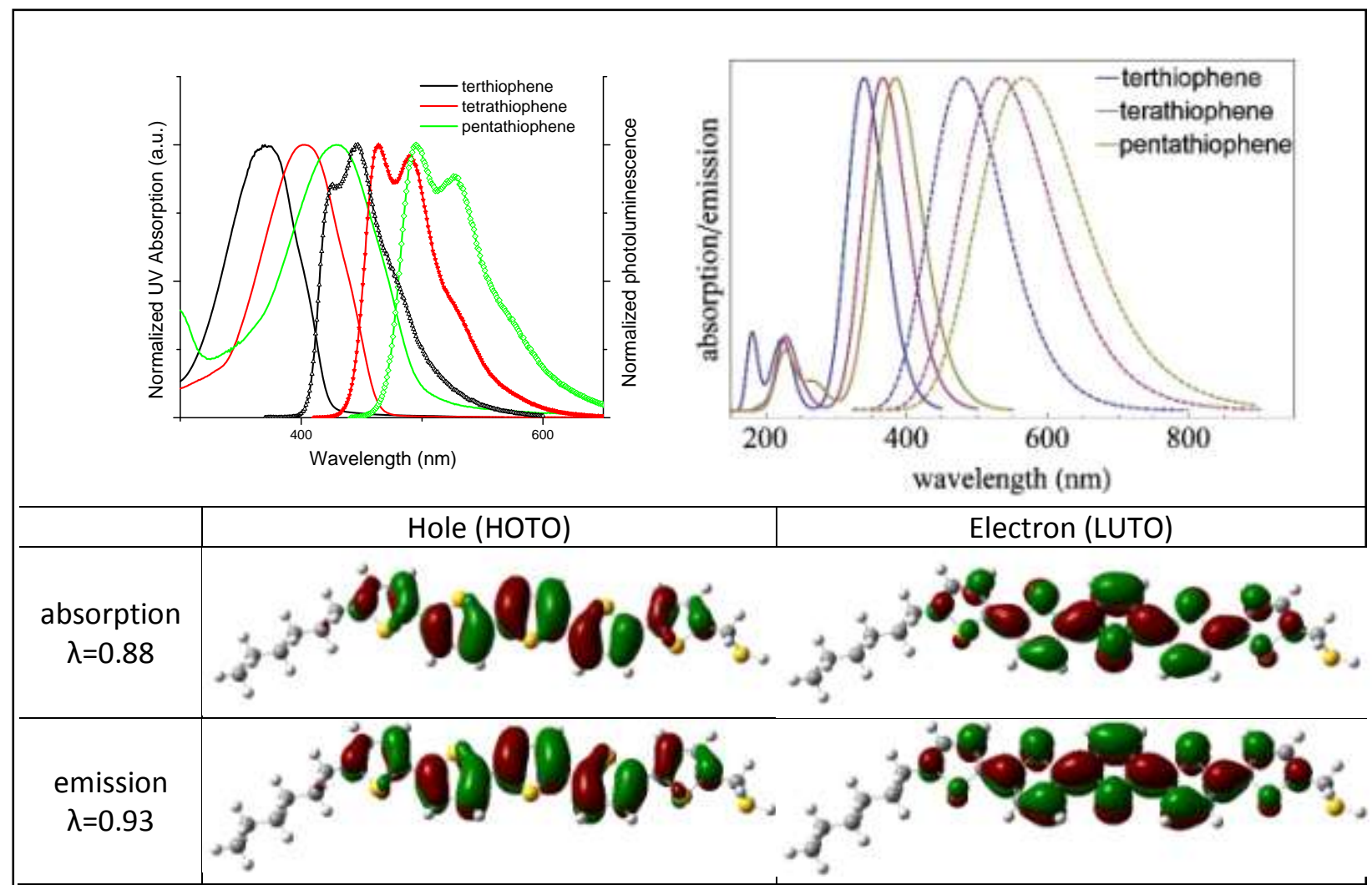

Figure 2. (Top) Normalized UV-Vis and fluorescence spectra of terthiophene,

tetrathiophene, and pentathiophene from experiments (left) and from simulations at $\omega B 97 X D / 6-31 G(d) / L R$ level (right). (Bottom) Natural transition orbitals describing the lowest vertical excitations and the lowest emission for monomer of thiolated pentathiophene.

The numbers $\lambda$ indicate the fraction of the NTO pair contribution to the given electronic excitation.

alternately twisted configurations are energetically favored for oligothiophene chains. In optimized excited-state geometries, the size of thiophene rings is slightly larger than the groundstate counterpart; the dihedral angle between rings turns to be about $180^{\circ}$ to form a roughly planar structure. The emission spectra are computed from the optimized geometry of the lowest excited state according to Kasha's rule. The simulation results of the normalized spectra of monomers are shown in the top-right panel of Figure 2, and compare well to their experimental counterparts.

Similar to experimental observations, longer thiophene chain results in larger extension

Table I. The maximum absorption and emission $\lambda_{\max }$ of thiolated oligothiophene monomer/dimer computed at theoretical level of $\omega B$ 97X-D/6-31G(d).

\begin{tabular}{|l|l|l|l|l|}
\hline $\begin{array}{l}\text { monomer/dimer } \\
\lambda_{\max }(\mathrm{nm})\end{array}$ & absorption(LR) & absorption(SS) & emission(LR) & emission(SS) \\
\hline terthiophene & $340 / 335$ & $331 / 326$ & $479 / 478$ & $435 / 448$ \\
\hline terathiophene & $366 / 359$ & $357 / 347$ & $532 / 530$ & $479 / 495$ \\
\hline pentathiophene & $384 / 386$ & $374 / 374$ & $565 / 562$ & $508 / 525$ \\
\hline
\end{tabular}


of the $\pi$-conjugation, as well as stronger solvent effect, observed as the red shift in both absorption and emission spectra. The maximum wavelengths $\lambda$ max are tabulated in Table I. The SS approach results in blue shifts compared to LR counterparts. The energy correction on emission spectra using the SS model is more noticeable than that of the LR approximation because the emission takes place in the solvent reaction field being equilibrium to the excitedstate charge distribution rather than to the ground-state counterpart. Therefore, the SS approach is more reliable in the simulation of fluorescent spectrum.

We have further visualized the natural transition orbitals (NTOs) [25], a compact orbital representation for electronic excitation, for the lowest vertical excitation and emission in order to compare the nature of different electronic transitions. The highest occupied transition orbital (HOTO) and the lowest unoccupied transition orbital (LUTO), corresponding the hole and the electron in an excitation, respectively, are shown in Figure 2. The visualized NTO pairs indicate that the lowest absorption and emission bands are attributed to similar delocalized $\pi-\pi^{*}$ electronic orbitals (see the example in Figure 2). It is worth mentioning that the frontier NTO pairs in solution obtained from both LR and SS approaches are visually identical to those in gas phase. These observations are valid for both ground- and excited-state geometries. The wave functions of electron and hole have approximately even and odd symmetries, respectively.

\section{Aggregated Species and Self-Assembly}

The comparison between the optical spectra of oligothiophenes in solution and as cast film is shown in Figure 2 and 3, which reveals structure-dependent optical properties of oligomers. The absorption spectra of terthiophene, tetrathiophene and pentathiophene in chloroform have a $\lambda$ max at 369, 402, and $430 \mathrm{~nm}$, respectively. The fluorescence spectra of terthiophene, tetrathiophene pentathiophene chloroform have vibronic features with two $\lambda$ max at 424, $446 \mathrm{~nm}, 462,490 \mathrm{~nm}$, and 495, $526 \mathrm{~nm}$, respectively. The as-cast films of three oligothiophenes exhibit strong red shifted PL spectra as compared to the solution counterparts, presumably due to the

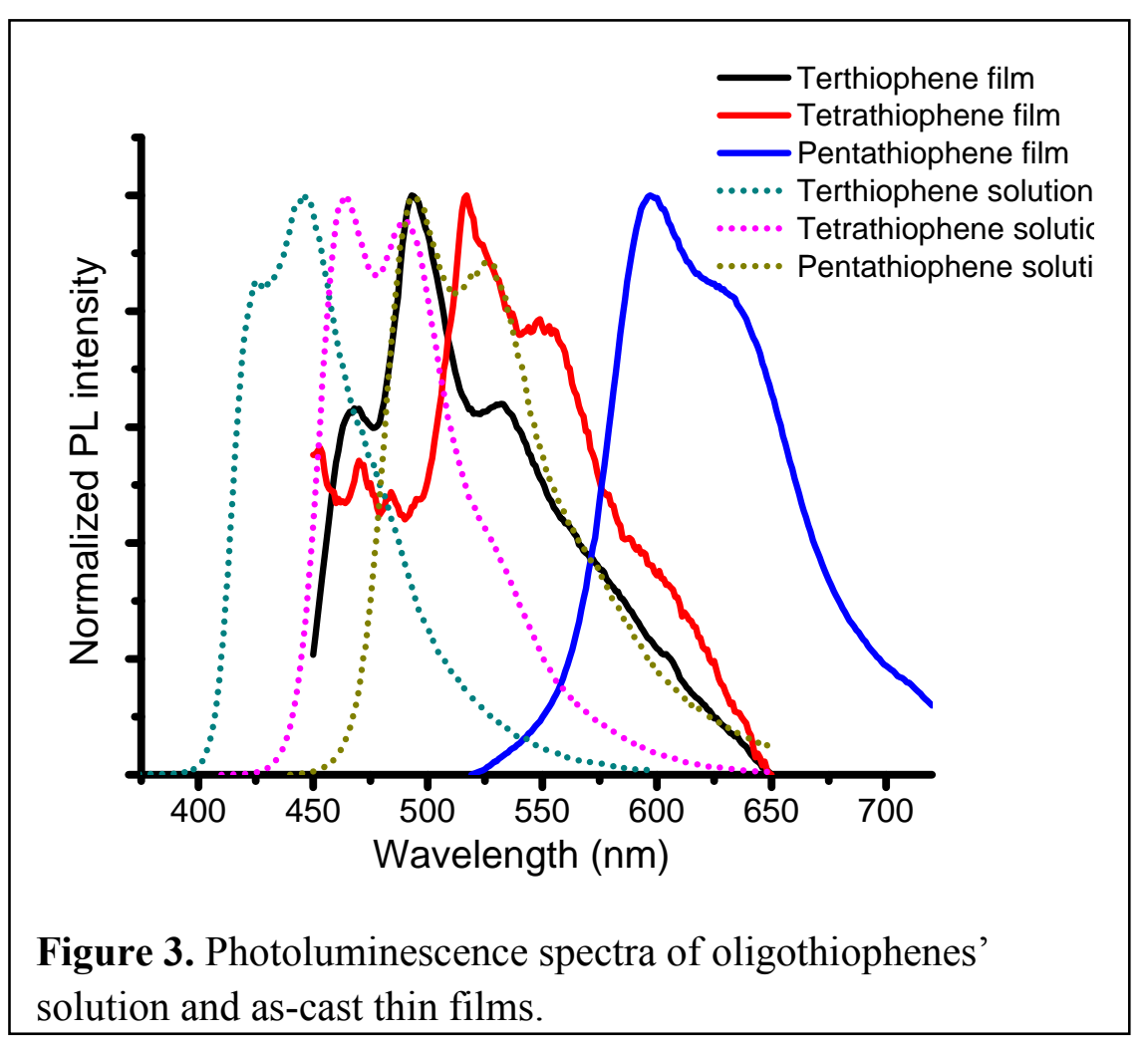


aggregation (stacking) between neighboring oligothiophene molecules. Our results show that the fluorescence spectra of terthiopehene, tetrathiophene and pentathiophene films have shoulder bands at 470, $500 \mathrm{~nm} ; 518,548 \mathrm{~nm}$; and 601, $628 \mathrm{~nm}$ respectively. In addition, we observe strong dependence of fluorescent properties of the films on excitation wavelength (see Supplemental Information, Fig S3, suggesting structural heterogeneity resulting from the aggregated (stacked) species [26].

To investigate molecular arrangement of the SAMs and dependence of their electronic and redox properties, oligothiophene molecules were attached to the $\mathrm{Au}(111)$ surface via $\mathrm{S}-\mathrm{Au}$ covalent bonding as described in Synthesis section. To our surprise, STM measurements reveal that only tetramer exhibits structural order at the molecular level, unlike the trimer and pentamer which show compacted globular structure on the Au surface, as shown in Figure 4. This contrast between the three oligomers can be rationalized as the pentamer has lower solubility than required for structuring these self-assemblies and inducing strong interaction between neighboring chromophores. The first look at the tetramers on single crystal $\mathrm{Au}(111)$ surface reveals extremely well aligned molecular layer with a periodic height variation of less than 1 $\mathrm{nm}$ and period of $0.5 \mathrm{~nm}$, suggesting a lay flat self-assembly along the long axis of the tetramer. It is also clear that there are several domains where tetramer self-assemblies are oriented in different directions, similar to polycrystalline facets (Figure $4 \mathrm{~b}$ ). We believe such highly ordered selfassembly is dominated by both $\pi-\pi$ interactions between chromophores

(a)

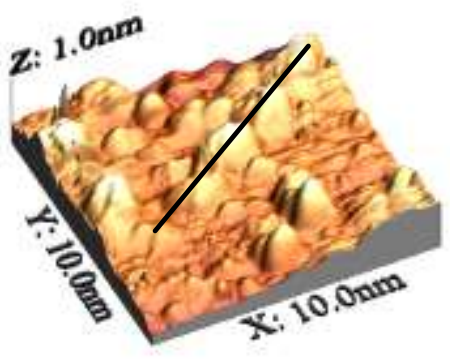

(b)

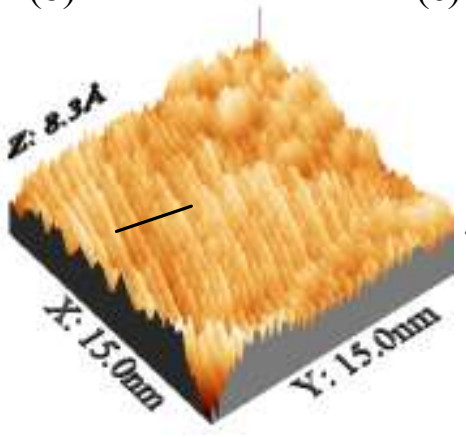

(c)

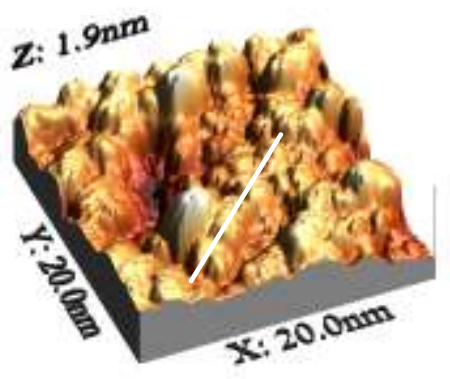

(d)

(e)

(f)
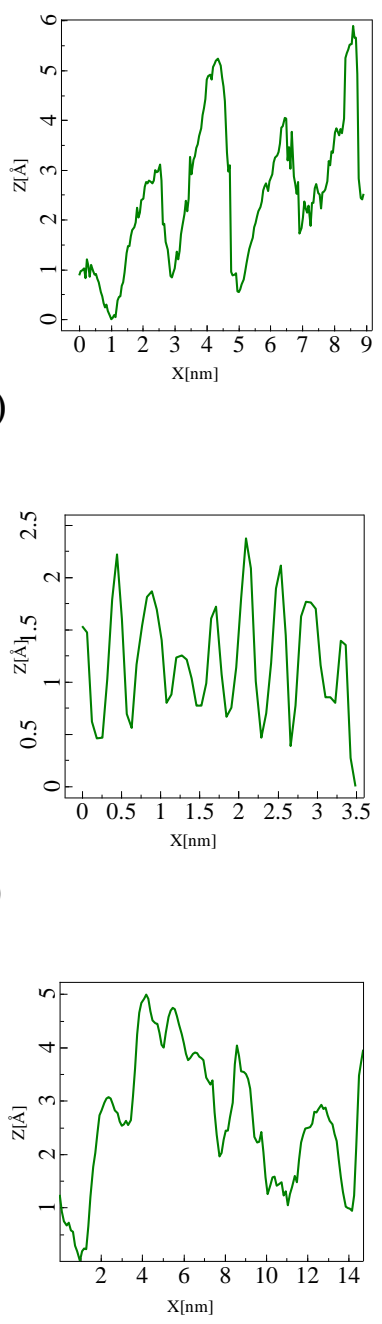

Figure 4. Topographic STM images $(3 \mathrm{~V}, 20 \mathrm{pA})$ of terthiophene (a), tetrathiophene (b), and pentathiophene (c). Corresponding depth profiles along the marked line on the surface of terthiophene (d), tetrathiophene (e) and pentathiophene (f) reveal the height and periodicity of the self-assembled thiophene monolayers. and surface interaction between tetramer and Au surface. The lay-flat configuration suggests 
that $\mathrm{Au}$ surface favors interaction with conjugated systems as it presents maximized contact with $\mathrm{Au}$ surface. Nevertheless, the majority of trimer and pentamer self-assemblies do not show ordered self-assembly, rather, the $\mathrm{Au}$ surface is covered by large amount of oligothiophene molecules which forms irregularly-shaped mounds of $\sim 2 \mathrm{~nm}$ in diameter and $<1 \mathrm{~nm}$ height.

To rationalize trends in the observed molecular assembly, we examine optimized dimer geometry of thiolated oligothiophenes which indeed shows different configurations with respect to the parity of the number of connected thiophenes. In the dimer geometry, the thiol group and the alkane chain are positioned on the same side of the oligothiophene plane (Figure 5) to minimize ground-state energy. Furthermore, because the adjacent thiophene units are twisted in different direction, viewing from the perspective of the projection on the approximate oligothiophene plane, the head and tail groups tend to be on the same side of the oligomer chain if the number of repeat units is odd, and on different sides of the chain for even number of thiophenes. Subsequently, the two molecules prefer to stay in a "face-to-face" configuration to maximize the intermolecular interaction and maximize $\pi$-orbital overlaps. Considering the mentioned relation between the side group relative orientation and the length parity of the oligomer, oligothiophenes with odd number of repeat units results in "parallel" molecular chains, in which the hexane and the thiol groups of one molecule face inward, i.e., being on the same side of the plane determined by the two oligothiophene axes, whereas those of the second molecule are oriented towards the other side of the plane due to the steric hindrance effect of the side groups (Figure 5, top). However, oligothiophenes with even number of repeat units adapt to "crossing" chain configuration, where the hexane and thiol groups of a molecule are oriented in opposite directions in relation to the molecular axis. This parity-related configuration is due to the alternate orientation of adjacent thiophenes in the oligothiophenes. Such dimer configuration remains valid in the optimized excited-state geometry. The distance between oligothiophene planes reduces in the optimized excited-state structure (3.2-3.5 $\AA$ ) compared to the ground-state counterpart (3.4-3.7 $\AA$ ). The binding and separation energies of the oligothiophene dimers are calculated at the DFT level (tabulated in Table SI in SI). Approximately, the binding energy linearly increases with the length of the oligomer. For benchmark purpose, separation energies of oligothiophene dimers without hexane and thiol groups have been computed to characterize the interaction between the $\pi$-conjugated structures. According to results of pure oligothiophenes, the binding strength (per thiophene pair) is around $0.19 \sim 0.23 \mathrm{eV}$ in dichloromethane solution can be found from the derivative of separation energy with respect to the conjugation length. It is also indicated that the thiol and hexane groups contribute about $0.46 \mathrm{eV}$ to the molecular binding in solution. 
When the monolayer selfassembles on a top of the single crystalline gold surface, it is almost impossible to measure the optical properties of such SAMs and determine variation in the UV-Vis and fluorescent spectra caused by aggregation. Instead we have compared dimer (Figure 5) and monomer spectra (Figure 2) obtained computationally. It is noticable that longer oligothiophene chain results in red shifts of both absorption and emission spectra of dimers (Figure 5). Compared to the monomers, small blue shift in dimer spectra has been calculated using the LR approach; the red shift of emission spectra computed by the SS model indicates weak state-dependent solvation energy correction for dimers. The $\lambda$ max of absorption and emission spectra of the oligothiophene dimers are also tabulated in Table I. The strongest absorption is due to the second excited state whereas the lowest excited state is responsible for the brightest emission in dichloromethane solution.

The computation result of

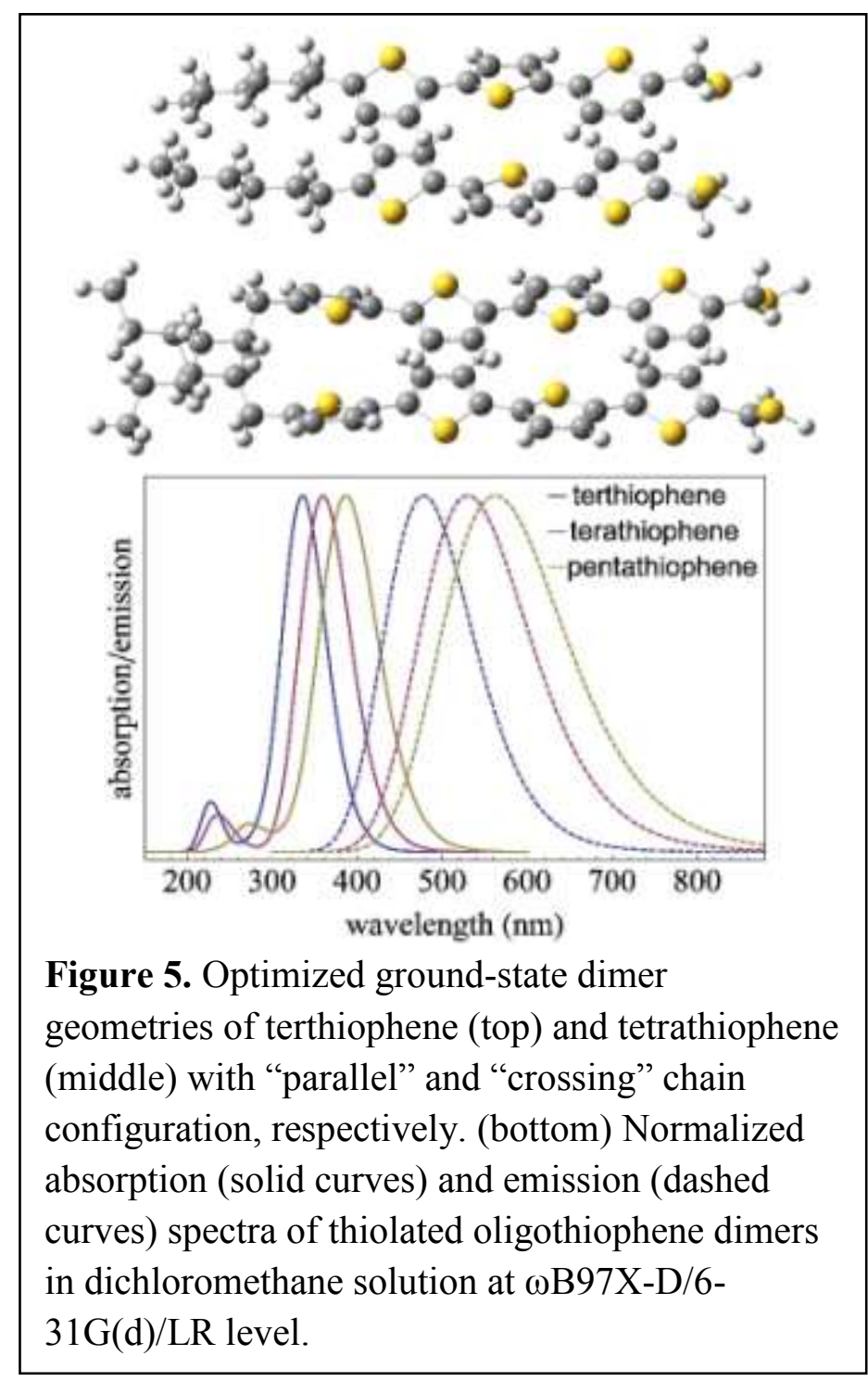
vertical excitations in oligothiophene dimers are summarized in Figure S2, where transition density matrices between the ground and excited states are visualized by contour plots [27]. As expected for all parallel dimer configurations, the lowest excited state is optically forbidden, whereas the second excited state is optically allowed (H-aggregate orientation). Above these states we observe several charge-transfer (CT) transitions as characterized by the two offdiagonal blocks in contour plots representing intermolecular transitions. The larger electronic dipole of a CT state considerably affects the solvent reaction field and makes this state sensitive to solvent relaxation. Therefore, the energies of CT states decrease substantially in the SS model compared to LR counterparts, whereas other excitonic states show blue shift (see Table SII in SI). The electronic transitions structure related to emission spectrum is also characterized by the contour plot of transition density in the bottom panel of Figure S2. The lowest excitation of a dimer in solution is confined within one molecule. Therefore, the lowest emission of dimer in solution is similar to that of the monomer, and similar fluorescent spectra (i.e., close energies and oscillator strengths) are obtained for both monomers and dimers in solution. 
Finally, we measure the redox potentials of the oligothiophenes in SAMs, spin cast films and solution. Of particular interest are tetrathiophene molecules as they self-assemble on the crystalline gold substrate and form well-ordered structures at the molecular level. The
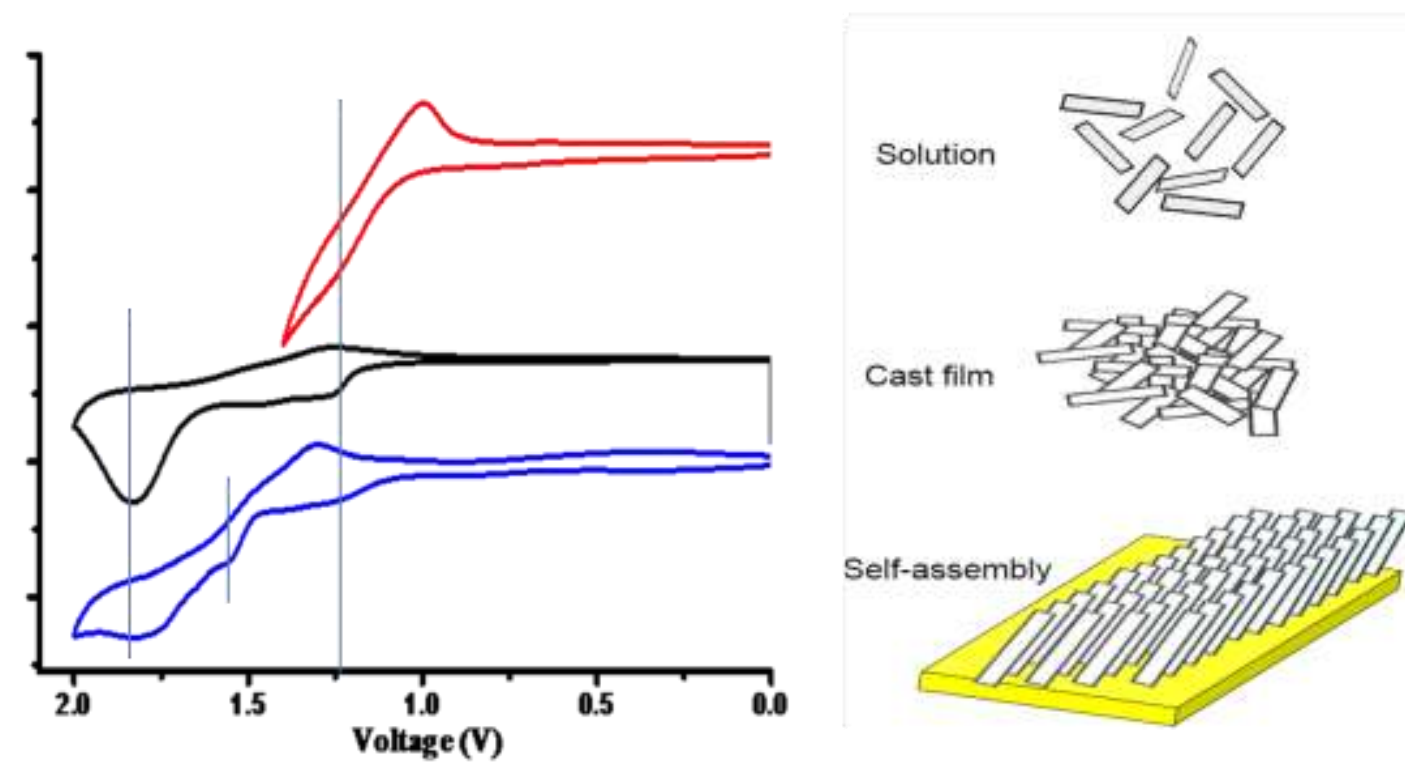

Figure 6. Cyclic voltammograms of tetrathiophene in solution, cast film and selfassembled monolayer thin film.

comparative studies of cyclic voltammograms between solution, cast film and self-assembled monolayer reveal several important features: only one oxidation peak $(1.16 \mathrm{~V})$ is observed in tetrathiophene solution, whereas two oxidation peaks $(1.17$, and $1.83 \mathrm{~V})$ are found when tetrathiophene is cast on Au electrode, and three oxidation peaks $(1.19,1.56$, and $1.80 \mathrm{~V})$ are seen in self-assembled monolayer with short and long range orders, as indicated in the STM micrograph. Such difference can be rationalized by the fact that the tetramer in solution allows only one radical cation per tetrathiophene molecule. Previous studies have shown that oxidation of polythiophene allows maximum charge density between 2-3 repeat units per radical cation [28]. The drop cast film has tetrathiophene molecules stacking on each another with strong electronic coupling between tetramers, which leads to a broadening of the electronic spectrum (Fig S4). Such interaction leads to the second oxidation peak which implies the formation of dications in cast film. The tetrathiophene monolayer self-assembly on gold surface exhibits three oxidation peaks, which typically requires extended conjugation and has only observed in few polythiophene derivatives [29]. A previous study show several quasi-reversible redox couples in oligothiophene functionalized dimethyldihydropyrenes and an increased applied potential lead to polymerization reaction [30]. The three oxidation peaks observed in tetrathiophene SAM mean that three electrons are extracted from the self-assembly: accommodation of three radical cations requires highly conjugated systems or ordered/strongly coupled electronic structure, consistent with the observed highly ordered tetrathiophene self-assembly. 


\section{Conclusions:}

We have performed synthesis and characterization of a series of oligothiophenes of different conjugation length with an alkyl head group and a thiol end group. These oligothiophenes exhibit structure-dependent optical and redox properties. In addition, only selected oligothiophenes show propensity for ordered self-assembly on single crystalline gold surfaces. In our studies, only tetrathiophene shows well-ordered structures at the molecular level resulting from a delicate balance between $\pi-\pi$ interaction between chromophores, hydrophobic interactions between alkyl head groups and $\mathrm{Au}-\mathrm{S}$ interaction between thiophene and the $\mathrm{Au}$ surface. The computational results from the oligothiophene dimers reveal an optimized interaction between oligothiophenes with odd number of thiophene repeat units (terthiophene and pentathiophene) as they show "parallel" molecular chains, in which both the hexyl and thiol groups are facing inward with respect to the $\pi$-conjugated backbone. Such arrangement allows maximized $\pi-\pi$, and hydrophobic interactions. The interaction between oligothiophenes with odd number repeat units overwhelms the interaction between oligothiophene and $\mathrm{Au}$ surface, hence leads to conglomerate of oligothiophene -- irregularly-shaped mounds of $\sim 2 \mathrm{~nm}$ in diameter and $<1 \mathrm{~nm}$ height. On the other hand, even number of thiophene repeat units such as tetrathiophene prefers dimer configuration similar to the "crossing" chains, where the hexyl and thiol groups are on the opposite side with respect to the molecular axis. This unfavorable orientation decreases $\pi-\pi$ interactions between tetrathiophenes whereas the covalent bonding between thiolated functional groups and Au surface prevails and allows homogeneous dispersion of tetrathiophene molecules in a highly ordered monolayer on the Au surface. The highly ordered hierarchical structure leads to strong electronic coupling and three redox couples in cyclic voltamogram that has not been observed in a simple oligothiophene system. Moreover, stabilization of multi-cations in ordered oligothiophene monolayers resembles that of the polythiophene and oligothiophene with stacked configuration. Overall, our study provides valuable insights into the evolution of structural, electronic and optical properties of oligothiophenes with the chain length and intermolecular coupling (from solution to selfassembled monolayers) which have promising implications for rational design of molecular electronic and energy devices with desired structure and properties.

\section{Acknowledgements:}

This work was performed in part at the Center for Integrated Nanotechnologies, a U.S. Department of Energy, Office of Basic Energy Sciences (DOE BES) user facility, and funded by the Los Alamos National Laboratory (LANL) Directed Research and Development program. The authors also acknowledge the LANL Institutional Computing (IC) Program for providing computational resources. LANL is operated by Los Alamos National Security, LLC, for the National Nuclear Security Administration of the U.S. DOE under contract DE-AC5206NA25396. 


\section{References:}

[1] (a) Barbarella, G.; Zambianchi, M.; Pudova, O.; Paladini, V.; Ventola, A.; Cipriani, F.; Gigli, G.; Cingolani, R.; Citro, G., Oligothiophene Isothiocyanates as a New Class of Fluorescent Markers for Biopolymers. Journal of the American Chemical Society 2001, 123 (47), 1160011607; (b) Melucci, M.; Zambianchi, M.; Favaretto, L.; Palermo, V.; Treossi, E.; Montalti, M.; Bonacchi, S.; Cavallini, M., Multicolor, large-area fluorescence sensing through oligothiopheneself-assembled monolayers. Chemical Communications 2011, 47 (6), 1689-1691; (c) Zhao, X. Y.; Schanze, K. S., Fluorescent ratiometric sensing of pyrophosphate via induced aggregation of a conjugated polyelectrolyte. Chemical Communications 2010, 46 (33), 6075-6077; (d) Crone, B.; Dodabalapur, A.; Gelperin, A.; Torsi, L.; Katz, H. E.; Lovinger, A. J.; Bao, Z., Electronic sensing of vapors with organic transistors. Applied Physics Letters 2001, 78 (15), 2229-2231. [2] (a) Mei, J.; Diao, Y.; Appleton, A. L.; Fang, L.; Bao, Z., Integrated Materials Design of Organic Semiconductors for Field-Effect Transistors. Journal of the American Chemical Society 2013, 135 (18), 6724-6746; (b) Reecht, G.; Bulou, H.; Scheurer, F.; Speisser, V.; Carrière, B.; Mathevet, F.; Schull, G., Oligothiophene Nanorings as Electron Resonators for Whispering Gallery Modes. Physical Review Letters 2013, 110 (5), 056802; (c) Cao, J.; Kampf, J. W.; Curtis, M. D., Synthesis and characterization of bis(3,4-ethylene-dioxythiophene)-(4,4 '-dialkyl-2,2 'bithiazole) co-oligomers for electronic applications. Chemistry of Materials 2003, 15 (2), 404411.

[3] (a) Antolini, L.; Tedesco, E.; Barbarella, G.; Favaretto, L.; Sotgiu, G.; Zambianchi, M.; Casarini, D.; Gigli, G.; Cingolani, R., Molecular packing and photoluminescence efficiency in odd-membered oligothiophene S,S-dioxides. Journal of the American Chemical Society 2000, 122 (37), 9006-9013; (b) Schanze, K. S.; Bergstedt, T. S.; Hauser, B. T., Photolithographic patterning of electroactive polymer films and electrochemically modulated optical diffraction gratings. Advanced Materials 1996, 8 (6), 531-\&; (c) Schenning, A. P. H. J.; Tsipis, A. C.; Meskers, S. C. J.; Beljonne, D.; Meijer, E. W.; Bredas, J. L., Electronic structure and optical properties of mixed phenylene vinylene/phenylene ethynylene conjugated oligomers. Chemistry of Materials 2002, 14 (3), 1362-1368.

[4] (a) Repp, J.; Liljeroth, P.; Meyer, G., Coherent electron-nuclear coupling in oligothiophene molecular wires. Nat Phys 2010, 6 (12), 975-979; (b) Bong, D.; Tam, I.; Breslow, R., Oligothiophene Isocyanides for Platinum-Based Molecular Electronic Applications. Journal of the American Chemical Society 2004, 126 (38), 11796-11797.

[5] Casado, J.; Ponce Ortiz, R.; Lopez Navarrete, J. T., Quinoidal oligothiophenes: new properties behind an unconventional electronic structure. Chemical Society Reviews 2012, 41 (17), 5672-5686.

[6] (a) Usta, H.; Facchetti, A.; Marks, T. J., n-Channel Semiconductor Materials Design for Organic Complementary Circuits. Accounts of Chemical Research 2011, 44 (7), 501-510; (b) Torsi, L.; Lovinger, A. J.; Crone, B.; Someya, T.; Dodabalapur, A.; Katz, H. E.; Gelperin, A., Correlation between Oligothiophene Thin Film Transistor Morphology and Vapor Responses. The Journal of Physical Chemistry B 2002, 106 (48), 12563-12568; (c) Murphy, A. R.; Fréchet, J. M. J.; Chang, P.; Lee, J.; Subramanian, V., Organic Thin Film Transistors from a Soluble Oligothiophene Derivative Containing Thermally Removable Solubilizing Groups. Journal of the American Chemical Society 2004, 126 (6), 1596-1597.

[7] (a) Servet, B.; Horowitz, G.; Ries, S.; Lagorsse, O.; Alnot, P.; Yassar, A.; Deloffre, F.; Srivastava, P.; Hajlaoui, R., Polymorphism and Charge Transport in Vacuum-Evaporated Sexithiophene Films. Chemistry of Materials 1994, 6 (10), 1809-1815; (b) Liedberg, B.; Yang, Z.; Engquist, I.; Wirde, M.; Gelius, U.; Gotz, G.; Bauerle, P.; Rummel, R. M.; Ziegler, C.; 
Gopel, W., Self-assembly of alpha-functionalized terthiophenes on gold. Journal of Physical Chemistry B 1997, 101 (31), 5951-5962.

[8] Kilina, S.; Batista, E. R.; Yang, P.; Tretiak, S.; Saxena, A.; Martin, R. L.; Smith, D. L., Electronic Structure of Self-Assembled Amorphous Polyfluorenes. ACS Nano 2008, 2(7), 13811388.

[9] Cygan, M. T.; Dunbar, T. D.; Arnold, J. J.; Bumm, L. A.; Shedlock, N. F.; Burgin, T. P.; Jones, L.; Allara, D. L.; Tour, J. M.; Weiss, P. S., Insertion, Conductivity, and Structures of Conjugated Organic Oligomers in Self-Assembled Alkanethiol Monolayers on Au $\{111\}$. Journal of the American Chemical Society 1998, 120 (12), 2721-2732.

[10] Cai, L.; Yao, Y.; Yang, J.; Price, D. W.; Tour, J. M., Chemical and Potential-Assisted Assembly of Thiolacetyl-Terminated Oligo(phenylene ethynylene)s on Gold Surfaces. Chemistry of Materials 2002, 14 (7), 2905-2909.

[11] Mei, J.; Kim, D. H.; Ayzner, A. L.; Toney, M. F.; Bao, Z., Siloxane-Terminated Solubilizing Side Chains: Bringing Conjugated Polymer Backbones Closer and Boosting Hole Mobilities in Thin-Film Transistors. Journal of the American Chemical Society 2011, 133 (50), 20130-20133.

[12] (a) Hendriksen, B. L. M.; Martin, F.; Qi, Y.; Mauldin, C.; Vukmirovic, N.; Ren, J.; Wormeester, H.; Katan, A. J.; Altoe, V.; Aloni, S.; Fréchet, J. M. J.; Wang, L.-W.; Salmeron, M., Electrical Transport Properties of Oligothiophene-Based Molecular Films Studied by Current Sensing Atomic Force Microscopy. Nano Letters 2011, 11 (10), 4107-4112; (b) Mattu, J. S.; Leach, G. W., Large Scale Crystallinity in Kinetically Stable Polythiophene-Based Langmuir-Blodgett Films. Journal of the American Chemical Society 2010, 132 (9), 3204-3210. [13] (a) Reeja-Jayan, B.; Koen, K. A.; Ono, R. J.; Vanden Bout, D. A.; Bielawski, C. W.; Manthiram, A., Oligomeric interface modifiers in hybrid polymer solar cell prototypes investigated by fluorescence voltage spectroscopy. Physical Chemistry Chemical Physics 2015, 17 (16), 10640-10647; (b) Planells, M.; Abate, A.; Snaith, H. J.; Robertson, N.; Planells, M.; Abate, A.; Snaith, H. J.; Robertson, N., Oligothiophene Interlayer Effect on Photocurrent Generation for Hybrid $\mathrm{TiO}<\mathrm{sub}>2</$ sub $>$ /P3HT Solar Cells. ACS Applied Materials \& Interfaces 2014, 6 (19), 17226-17235; (c) Nie, W.; Gupta, G.; Crone, B. K.; Liu, F.; Smith, D. L.; Ruden, P. P.; Kuo, C.-Y.; Tsai, H.; Wang, H.-L.; Li, H.; Tretiak, S.; Mohite, A. D., Semiconductors: Interface Design Principles for High-Performance Organic Semiconductor Devices (Adv. Sci. 6/2015). Advanced Science 2015, 2 (6), n/a-n/a.

[14] Frisch, M. J. T., G. W.; Schlegel, H. B.; Scuseria, G. E.; Robb, M. A.; Cheeseman, J. R.; Scalmani, G.; Barone, V.; Mennucci, B.; Petersson, G. A.; Nakatsuji, H.; Caricato, M.; Li, X.; Hratchian, H. P.; Izmaylov, A. F.; Bloino, J.; Zheng, G.; Sonnenberg, J. L.; Hada, M.; Ehara, M.; Toyota, K.; Fukuda, R.; Hasegawa, J.; Ishida, M.; Nakajima, T.; Honda, Y.; Kitao, O.; Nakai, H.; Vreven, T.; Montgomery, J. A., Jr.; Peralta, J. E.; Ogliaro, F.; Bearpark, M.; Heyd, J. J.; Brothers, E.; Kudin, K. N.; Staroverov, V. N.; Kobayashi, R.; Normand, J.; Raghavachari, K.; Rendell, A.; Burant, J. C.; Iyengar, S. S.; Tomasi, J.; Cossi, M.; Rega, N.; Millam, J. M.; Klene, M.; Knox, J. E.; Cross, J. B.; Bakken, V.; Adamo, C.; Jaramillo, J.; Gomperts, R.; Stratmann, R. E.; Yazyev, O.; Austin, A. J.; Cammi, R.; Pomelli, C.; Ochterski, J. W.; Martin, R. L.; Morokuma, K.; Zakrzewski, V. G.; Voth, G. A.; Salvador, P.; Dannenberg, J. J.; Dapprich, S.; Daniels, A. D.; Farkas, Ö.; Foresman, J. B.; Ortiz, J. V.; Cioslowski, J.; Fox, D. J. , Gaussian 09, Revision D.01, Gaussian, Inc., Wallingford CT, 2009.

[15] Chai, J.-D.; Head-Gordon, M., Long-range corrected hybrid density functionals with damped atom-atom dispersion corrections. Physical Chemistry Chemical Physics 2008, 10 (44), 6615-6620. 
[16] Grimme, S., Semiempirical GGA-type density functional constructed with a long-range dispersion correction. Journal of Computational Chemistry 2006, 27 (15), 1787-1799.

[17] (a) Li, H.; Nieman, R.; Aquino, A. J. A.; Lischka, H.; Tretiak, S., Comparison of LCTDDFT and ADC(2) Methods in Computations of Bright and Charge Transfer States in Stacked Oligothiophenes. Journal of Chemical Theory and Computation 2014, 10 (8), 3280-3289; (b) Magyar, R. J.; Tretiak, S., Dependence of Spurious Charge-Transfer Excited States on Orbital Exchange in TDDFT: Large Molecules and Clusters. Journal of Chemical Theory and Computation 2007, 3 (3), 976-987.

[18] (a) Schirmer, J., Beyond the random-phase approximation: A new approximation scheme for the polarization propagator. Physical Review A 1982, 26 (5), 2395-2416; (b) Trofimov, A. B.; Schirmer, J., An efficient polarization propagator approach to valence electron excitation spectra. Journal of Physics B: Atomic, Molecular and Optical Physics 1995, 28 (12), 2299; (c) Trofimov, A. B.; Krivdina, I. L.; Weller, J.; Schirmer, J., Algebraic-diagrammatic construction propagator approach to molecular response properties. Chemical Physics 2006, 329 (1-3), 1-10.

[19] Stephens, P. J.; Harada, N., ECD cotton effect approximated by the Gaussian curve and other methods. Chirality 2010, 22 (2), 229-233.

[20] (a) Barone, V.; Cossi, M., Quantum Calculation of Molecular Energies and Energy Gradients in Solution by a Conductor Solvent Model. The Journal of Physical Chemistry A 1998, 102 (11), 1995-2001; (b) Cossi, M.; Rega, N.; Scalmani, G.; Barone, V., Energies, structures, and electronic properties of molecules in solution with the C-PCM solvation model. Journal of Computational Chemistry 2003, 24 (6), 669-681; (c) Tomasi, J.; Mennucci, B.; Cammi, R., Quantum Mechanical Continuum Solvation Models. Chemical Reviews 2005, 105 (8), 29993094.

[21] (a) Cammi, R.; Corni, S.; Mennucci, B.; Tomasi, J., Electronic excitation energies of molecules in solution: State specific and linear response methods for nonequilibrium continuum solvation models. The Journal of Chemical Physics 2005, 122 (10), 104513; (b) Improta, R.; Barone, V.; Scalmani, G.; Frisch, M. J., A state-specific polarizable continuum model time dependent density functional theory method for excited state calculations in solution. The Journal of Chemical Physics 2006, 125 (5), 054103; (c) Improta, R.; Scalmani, G.; Frisch, M. J.; Barone, V., Toward effective and reliable fluorescence energies in solution by a new state specific polarizable continuum model time-dependent density functional theory approach. The Journal of Chemical Physics 2007, 127 (7), 074504.

[22] Magyar, R. j.; Tretiak, S.; Gao, Y.; Wang, H.-L.; Shreve, A. P., A Joint Theoretical and Experimental Study of Phenylene-Acetylene Molecular Wires. Chem. Phys. Lett. 2005, 401(1-3), 149-156.

[23] (a) Padmanaban, G.; Ramakrishnan, S., Conjugation Length Control in Soluble Poly[2methoxy-5-((2‘-ethylhexyl)oxy)-1,4-phenylenevinylene] (MEHPPV): Synthesis, Optical Properties, and Energy Transfer. Journal of the American Chemical Society 2000, 122 (10), 2244-2251; (b) Bredas, J. L.; Silbey, R.; Boudreaux, D. S.; Chance, R. R., Chain-length dependence of electronic and electrochemical properties of conjugated systems: polyacetylene, polyphenylene, polythiophene, and polypyrrole. Journal of the American Chemical Society 1983, 105 (22), 6555-6559; (c) Yamaguchi, Y.; Matsubara, Y.; Ochi, T.; Wakamiya, T.; Yoshida, Z.-i., How the $\pi$ Conjugation Length Affects the Fluorescence Emission Efficiency. Journal of the American Chemical Society 2008, 130 (42), 13867-13869.

[24] (a) Kim, D. Y.; Grey, J. K.; Barbara, P. F., A detailed single molecule spectroscopy study of the vibronic states and energy transfer pathways of the conjugated polymer MEH-PPV. Synthetic Metals 2006, 156 (2-4), 336-345; (b) Barbara, P. F.; Gesquiere, A. J.; Park, S.-J.; Lee, 
Y. J., Single-Molecule Spectroscopy of Conjugated Polymers. Accounts of Chemical Research 2005, 38 (7), 602-610; (c) Huser, T.; Yan, M.; Rothberg, L. J., Single chain spectroscopy of conformational dependence of conjugated polymer photophysics. Proceedings of the National Academy of Sciences 2000, 97 (21), 11187-11191.

[25] Martin, R. L., Natural transition orbitals. The Journal of Chemical Physics 2003, 118 (11), 4775-4777.

[26] (a) Noriega, R.; Rivnay, J.; Vandewal, K.; Koch, F. P. V.; Stingelin, N.; Smith, P.; Toney, M. F.; Salleo, A., A general relationship between disorder, aggregation and charge transport in conjugated polymers. Nat Mater 2013, 12 (11), 1038-1044; (b) Spano, F. C., Absorption and emission in oligo-phenylene vinylene nanoaggregates: The role of disorder and structural defects. Journal of Chemical Physics 2002, 116 (13), 5877-5891; (c) Spano, F. C., EXCITONS IN CONJUGATED OLIGOMER AGGREGATES, FILMS, AND CRYSTALS. Annual Review of Physical Chemistry 2006, 57 (1), 217-243.

[27] (a) Tretiak, S.; Zhang, W. M.; Chernyak, V.; Mukamel, S., Excitonic couplings and electronic coherence in bridged naphthalene dimers. Proceedings of the National Academy of Sciences of the United States of America 1999, 96 (23), 13003-13008; (b) Schulz, M.; Tretiak, S.; Chernyak, V.; Mukamel, S., Size Scaling of Third-Order Off-Resonant Polarizabilities. Electronic Coherence in Organic Oligomers. Journal of the American Chemical Society 2000, $122(3), 452-459$.

[28] Yamamoto, T., Molecular assembly and properties of polythiophenes. NPG Asia Mater 2010, 2, 54-60.

[29] (a) Demanze, F.; Yassar, A.; Garnier, F., Push-pull substituted polythiophenes: towards charge confinement in molecular quantum wells. Advanced Materials 1995, 7 (11), 907-910; (b) Chen, X.; Inganäs, O., Three-Step Redox in Polythiophenes: Evidence from Electrochemistry at an Ultramicroelectrode. The Journal of Physical Chemistry 1996, 100 (37), 15202-15206.

[30] Robinson, S. G.; Lonergan, M. C.; Mitchell, R. H., Oligothiophene Functionalized Dimethyldihydropyrenes II: Electrochemical and Conductive Properties. The Journal of Organic Chemistry 2009, 74 (17), 6606-6614. 


\section{Graphical Abstract}

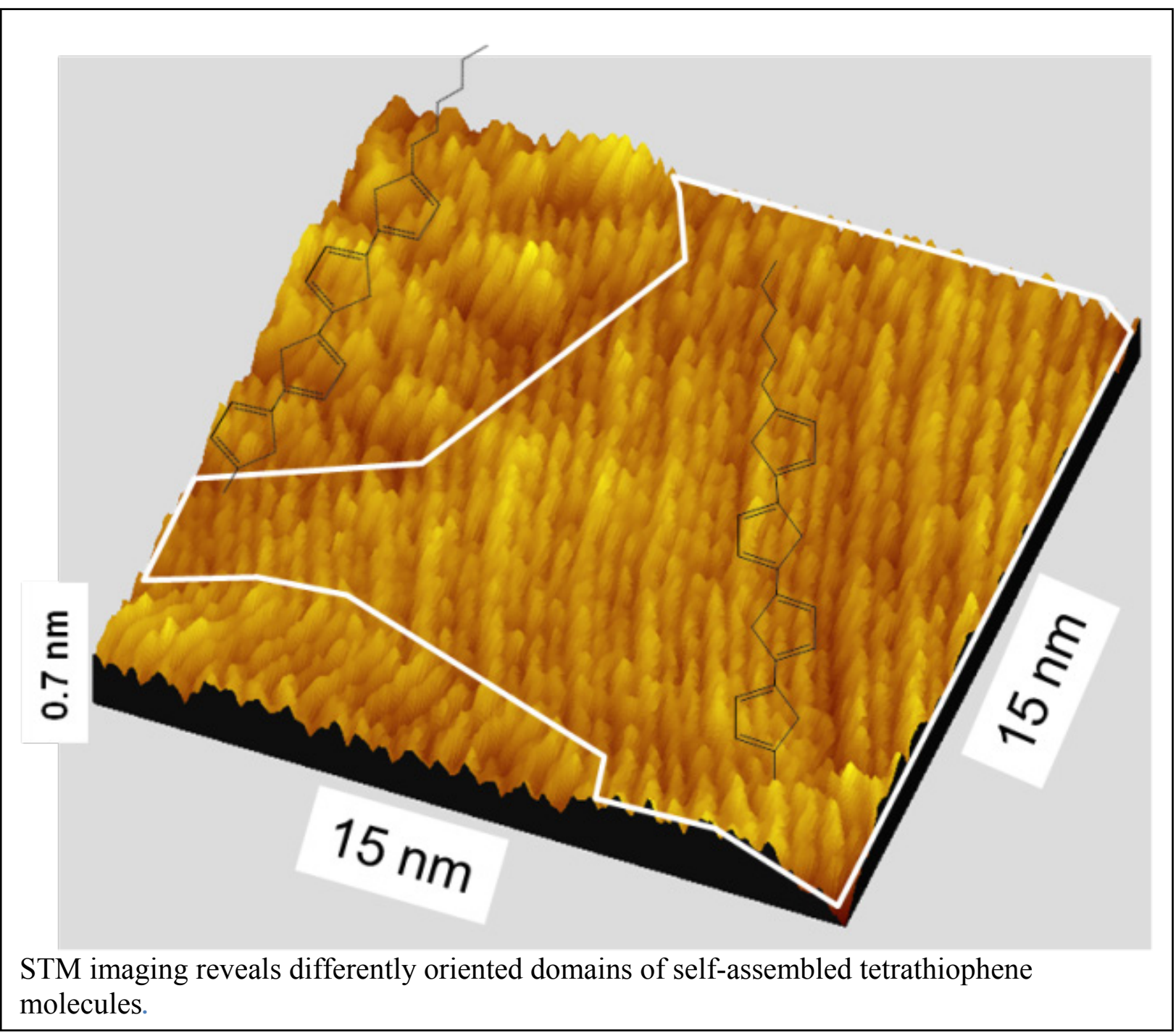

